\title{
Optimization of Power System Stabilizer for Multi- Machine Power System using Invasive Weed Optimization Algorithm
}

\author{
Ashik Ahmed \\ Assistant Professor \\ EEE Department \\ Islamic University of Technology, Gazipur, \\ Bangladesh.
}

\author{
B. M. Ruhul Amin \\ Lecturer \\ EEE Department \\ Bangladesh University of Business and Technology
}

\begin{abstract}
In this paper, an evolutionary algorithm-Invasive Weed Optimization (IWO) based power system stabilizer (PSS) is proposed for multi-machine power system. IWO is a derivative-free real parameter optimization technique that mimics the ecological behavior of colonizing weeds. Owing to its superior performance in comparison with many other existing meta-heuristics, it has used to search for optimal settings of PSS parameters. Eigen-value based objective function is considered to enhance system damping of electromechanical mode. The performance of proposed IWObased PSS is tested and demonstrated under different loading conditions and disturbances for a four machine example power system. The Eigen value analysis and non-linear simulation results prove the effectiveness of the proposed IWO-based PSS design. The robustness of the design method is confirmed by testing the IWO based PSS performance under varying load conditions.
\end{abstract}

\section{General Terms}

Power system stability, Multi-machine system, Optimization.

\section{Keywords}

PSS design, Invasive Weed Optimization, Dynamic stability.

\section{INTRODUCTION}

Modern power industry has been trying with newer approaches to maximize power transfer among different areas in a stable manner. The transmission networks are usually overdesigned to keep the operation of the overall system within safe limit. This safety measure is taken due to the constraints of thermal limit, rotor angle stability limit and voltage stability limit [1]. A serious threat to the safe operation of power system is the presence of low frequency oscillation among the interconnected group of generators. One cause of these oscillations is the presence of weak tie lines among two large systems. Oscillations residing within a plant are termed as local or plant mode of oscillations whereas those among the interconnected systems are named as interarea mode of oscillations. These modes normally have a frequency range of $0.7-2.0 \mathrm{~Hz}$ and $0.1-0.8 \mathrm{~Hz}$ respectively [2]. Considerable effort has been made to clarify the impact of Power System Stabilizer (PSS) in damping low frequency oscillation and thus improve the small signal stability of power system [3]. PSS is found to be very effective and economic and that has ensured wide use of it by the utilities. Different modern control theory based approaches have been applied to PSS design problem. These contain optimal control, fuzzy \& neuro-fuzzy control, variable structure control and adaptive control [4-7]. It is shown in [8] that appropriate selection of conventional PSS parameters result in satisfactory performance under system disturbances. Two basic approaches are adopted for the design purpose of PSS, namely, sequential tuning and simultaneous tuning. In sequential design of PSS one electromechanical mode considered for damping at a time among many available modes [9]. The optimal parameters designed for a certain mode can create adverse effect on some other modes of the system and the overall performance may not be optimal. The simultaneous tuning of PSS problem is formulated as nonlinear non differentiable optimization problem [10] which is very hard to solve using traditionally differentiable optimization algorithms. Different random exploration techniques like Tabu Search (TS), Simulated Annealing (SA), Genetic Algorithm (GA), Evolutionary Programming (EP) has been used [11-15] to optimize the PSS parameters. The techniques have gained acceptance because of their ability and effectiveness of searching an optimal solution in a problem space. Main objective is to modify the damping ratio and the damping factor of the lightly damped or un-damped electromechanical modes. It is found that taking just one of the objectives into account yields an unacceptable result for other objectives. In their method, just the unstable or lightly damped oscillation modes are of main concern. A frequency domain based approach [16] is found to be more appropriate where a group of objectives are listed and GA is used to optimize them. Although GA is found to be very satisfactory in searching global or near global optimal result of the problem, it needs a very long run time that may vary depending on the size of the system under study. Recently a derivative- free, meta-heuristic optimization algorithm named as Invasive Weed Optimization (IWO) is proposed [17] which mimic the ecological behavior of the colonizing weeds. Since then IWO has been successfully utilized in different practical optimization problems like optimization and tuning of a robust controller [17], optimal positioning of piezoelectric actuators [18], design of E-shaped MIMO antenna [20], designing of encoding sequences for DNA computing [21], developing a recommender system [22] and studying electricity market dynamics [23].

In this paper this recently developed IWO is used for designing PSSs with optimized performance in multi-machine power system. The PSS design problem is formulated as optimization problem with constraints and an eigenvalue based objective function. An IWO algorithm is then utilized to solve the constrained optimization problem. To investigate effectiveness of the proposed method a four machine power system [1] has been considered. Eigenvalue analysis and nonlinear time domain simulations have been performed to 
justify the efficacy of the proposed PSSs under different loading conditions.

\section{PROBLEM STATEMENT}

\subsection{System model and PSS structure}

A multi-machine power system can be modeled by a system of nonlinear equations of the form

$$
\dot{X}=f(X, U)
$$

where $\mathrm{X}$ is the vector of state variables and $\mathrm{U}$ is the vector of input variables. In this work a two axis model [24] given in the Appendix is used for the simulation purpose. In PSS design problem the linearized model of the power system around an operating point is usually employed. Therefore for an $\mathbf{m}$ machine npss stabilizer power system the state equation of Eq. (1) becomes

$$
\Delta \dot{X}=A \Delta X+B \Delta U
$$

Where, $\mathrm{A}$ is $4 m \mathrm{X} 4 m$ state matrix, $\Delta X$ is $4 m X 1$ state vector, B is $4 m X n p s s$ input matrix, $\Delta U$ is $n p s s X 1$ input vector. Matrix A equals $\frac{\partial f}{\partial x}$, matrix B equals $\frac{\partial f}{\partial u}$; both evaluated at $x_{0}$ which is the operating point around which the system is linearized.

The function of a PSS is to compensate the phase lag between the exciter input and the machine electrical torque by generating appropriate torque on the rotor of the machine. In this paper widely used speed based PSS design is considered where the stabilizing signal is assumed to be proportional to the speed. The structure of PSS is shown in Fig. 1. It consists of a gain block with gain $K_{p s s i}$, a signal washout block and two-stage phase compensation blocks. Hence, the transfer function of the $i$ th PSS is given by:

$$
U_{p s s, i}=K_{p s s, i} \frac{s T_{w i}\left(1+s T_{1 i}\right)\left(1+s T_{3 i}\right)}{1+s T_{w i}\left(1+s T_{2 i}\right)\left(1+s T_{4 i}\right)} \Delta \omega_{i}
$$

Where, $\Delta \omega_{i}$ and $U_{p s s i}$ are the deviation of synchronous speed and the stabilizer control signal which is added to the excitation system reference. The signal washout block time constant $T_{w i}$ allows the signal associated with oscillations in rotor speed to pass unchanged, and restricts the steady state changes to modify the terminal voltages. From the view of the washout function, the value of $T w$ is generally not critical and may be in the range of 0.5 to 20 seconds [23]. In this paper, it is fixed to $10 \mathrm{~s}$. The phase compensation block with time constants $T_{1}, T_{2}$ and $T_{3}, T_{4}$ supplies the required phase-lead to compensate for the phase lag between input and the output signals. The five PSS parameters consisting of the four time constants $T_{1}$ to $T_{4}$ and the gain $K_{p s s}$ need to be optimally chosen for each generator to guarantee optimal system performance under various system configurations and system loadings to ensure the robustness of the technique.

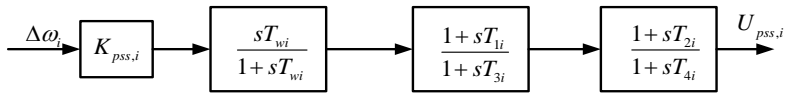

Fig.1: Power system stabilizer structure

\subsection{Objective function}

To select the best stabilizer parameters that enhance the power system dynamic performance the most, an eigenvalue based objective function is considered as given in Eq. 4.

$$
\mathrm{J}=-\min (\text { real (eigenvalues) / abs (eigenvalues ) ) }
$$

So the objective here is to maximize the minimum of the damping ratio for a certain parameter set. Maximizing the damping ratio will certainly help the cause of improving the system overall damping. The problem constraints are the stabilizer parameter bounds in this case. Therefore, the design problem can be formulated as the following optimization problem:

$$
\text { Maximize } \mathbf{J}
$$

Subject to,

$$
\begin{gathered}
K_{i}^{\min } \leq K_{i} \leq K_{i}^{\max } \\
T_{1 i}^{\min } \leq T_{1 i} \leq T_{1 i}^{\max } \\
T_{2 i}^{\min } \leq T_{2 i} \leq T_{2 i}^{\max } \\
T_{3 i}^{\text {min }} \leq T_{3 i} \leq T_{3 i}^{\max } \\
T_{4 i}^{\min } \leq T_{4 i} \leq T_{4 i}^{\max }
\end{gathered}
$$

where $\mathrm{i}$ is the number of stabilizers considered and $\mathrm{J}$ is the objective function defined in equation (4). The proposed approach will employ Invasive Weed optimizer (IWO) to search for the optimum parameter settings of the given stabilizers.

\section{INVASIVE WEED OPTIMIZATION}

Invasive Weed Optimization is a bio-inspired numerical stochastic optimization algorithm that simply simulates natural behavior of weeds in colonizing and finding suitable place for growth and reproduction. Some of the distinctive properties of IWO in comparison with other evolutionary algorithms are the way of reproduction, spatial dispersal, and competitive exclusion [17].

The IWO process begins with initializing a population. That is, a population of initial solutions is randomly generated over the solution space. Then members of the population produce seeds depending on their comparative fitness in the population. In other words, the number of seeds for each member varies linearly between $S_{\min }$ for the worst member and $S_{\max }$ for the best member. These seeds are then randomly scattered over the search space by normally distributed random numbers with mean equal to zero and an adaptive standard deviation. The equation for determining the standard deviation (SD) for each generation is presented as:

$\sigma_{\text {iter }}=\frac{\left(\text { iter }_{\max }-\text { iter }\right)^{n}}{\left(\text { iter }_{\max }\right)^{n}}\left(\sigma_{\text {initial }}-\sigma_{\text {final }}\right)+\sigma_{\text {final }}$

where iter $_{\max }$ is the maximum number of iterations, $\sigma_{\text {iter }}$ is the $\mathrm{SD}$ at the current iteration and $n$ is the nonlinear modulation index. The produced seeds, accompanied by their parents are considered as the potential solutions for the next generation. 
Finally, a competitive exclusion is conducted in the algorithm, i.e. after a number of iterations the population reaches its maximum, and an elimination mechanism is adopted. The seeds and their parents are ranked together and those with better fitness survive and become reproductive. A flow chart describing the IWO algorithm is presented in Fig. 2.

\subsection{Selection of control parameters}

The convergence of the algorithm depends on the selection of three parameters, the initial $\mathrm{SD}$, ' $\sigma_{\text {initial }}$ ', the final $\mathrm{SD}$, ' $\sigma_{\text {final }}$ ' and the nonlinear modulation index ' $n$ '. These three must be tuned in such a way that the proper value of SD in every iteration is achieved, according to (11). High value of $\sigma_{\text {initial }}$ helps the algorithm search the whole search space aggressively whereas a finer optimum solution can be achieved by decreasing the parameter $\sigma_{\text {final }}$. The impact of the nonlinear modulation index on the performance of the optimizer is shown in [17]. It is shown that the best value of ' $n$ ' in Eq. (11) is 3 [24] if the SD for each iteration is expressed by (11). Maximum and minimum numbers of seeds are the other two criteria than influence the performance of the algorithm. A value between 3 and 5 for the maximum and 0 for the minimum yields good results as found in many examples [24]. Finally the maximum number of plants should also be chosen for IWO. It was found that a value between 10 and 20 gives excellent performance for many problems [24].

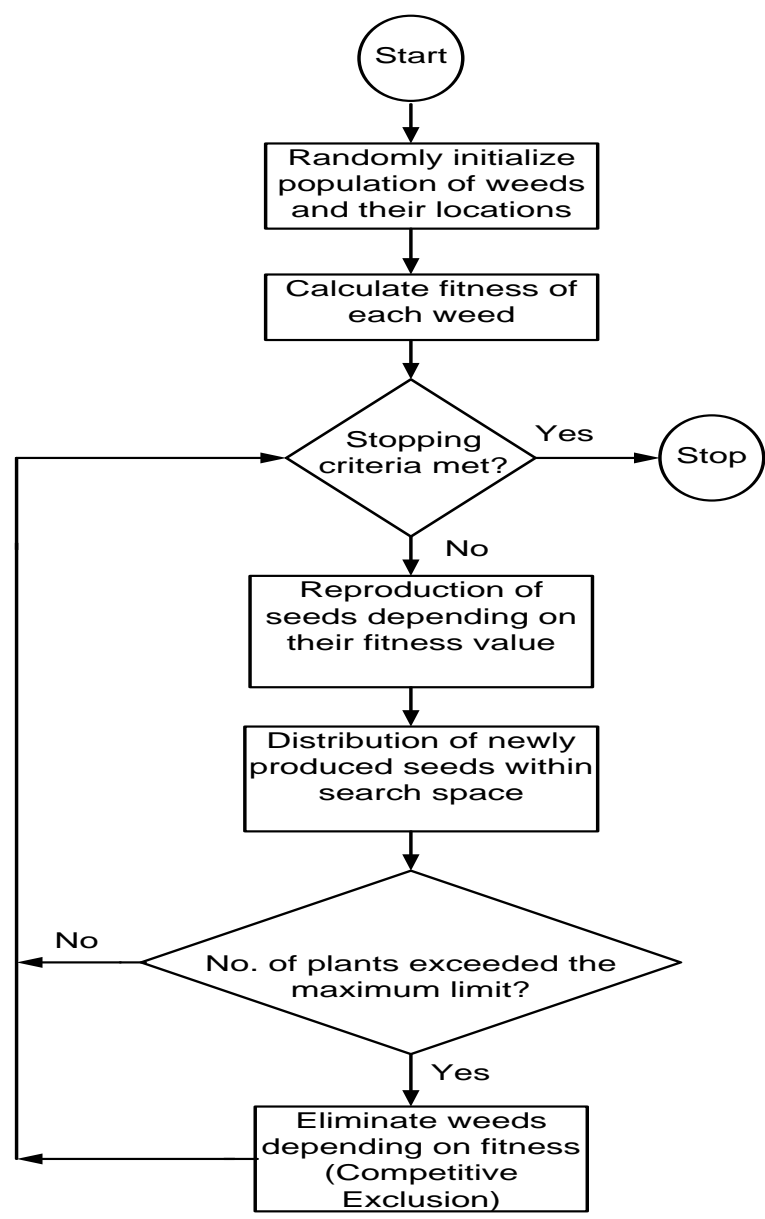

Fig.2: Invasive Weed Optimization algorithm flowchart

\section{SIMULATION RESULTS}

The single-line diagram of two-area, 4-machine test system, as shown in Figure 3, is used to examine both local and inter area oscillations control problems. The system consists of four generators, G1 and G2 for area 1 and G3 and G4 for area 2. The system loads L7 and L9 are present at buses 7 and 9 respectively. Detail of the system data can be obtained from [2]. For illustration and comparison purpose all four generators assumed to have PSSs installed. It is also decided that all four time constants for each PSS with two lead-lag blocks and the PSS gain will be tuned to obtain the optimum value. So the number of parameters to be optimized for this work is 20. The maximum and minimum ranges for the gains and different time constants for optimization purpose are set at [0-50] and [0.01-1.0] respectively. The IWO algorithm is then applied to find out the optimized value of parameters for the objective function in (4). The final values of the optimized parameters are provided in Table I.

\subsection{Eigenvalue analysis and nonlinear simulation results}

Three different cases as nominal, light and heavy loading are considered to prove the effectiveness and robustness of the proposed method over a wide range of loading conditions. The system loading levels at these cases are given in Table II. The IWO algorithm runs several times and then optimal set of PSS parameters are selected. The eigenvalues and the damping ratio of electromechanical modes without PSS is given in Table III and the eigenvalues and the damping ratio of electromechanical modes with PSS is given in Table IV. In table III we observe some eigenvalues in the right side of splane and thus obtain negative damping ratio which causes severe oscillation in the system.

\section{TABLES AND FIGURES}

Table 1: Optimal PSS parameters with objective functions

\begin{tabular}{|c|c|c|c|c|c|c|}
\hline \multirow[t]{2}{*}{ Gen } & & & & & & Load \\
\hline & $\mathrm{K}$ & $\mathrm{T}_{1}$ & $\mathrm{~T}_{2}$ & $\mathrm{~T}_{3}$ & $\mathrm{~T}_{4}$ & \\
\hline $\mathrm{G}_{1}$ & 45.6522 & 0.0832 & 0.3830 & 0.8059 & 0.0100 & \multirow{4}{*}{ Nominal } \\
\hline $\mathrm{G}_{2}$ & 11.5510 & 0.2823 & 0.3637 & 0.7101 & 0.5891 & \\
\hline $\mathrm{G}_{3}$ & 40.7216 & 0.1494 & 0.0580 & 0.3501 & 0.4679 & \\
\hline $\mathrm{G}_{4}$ & 32.6851 & 0.2146 & 0.6437 & 0.4617 & 0.0100 & \\
\hline $\mathrm{G}_{1}$ & 43.7651 & 0.0933 & 0.4003 & 0.7565 & 0.0100 & \multirow{4}{*}{ Light } \\
\hline $\mathrm{G}_{2}$ & 21.7023 & 0.4274 & 0.6020 & 0.6774 & 0.5842 & \\
\hline $\mathrm{G}_{3}$ & 31.0260 & 0.3345 & 0.8982 & 0.9922 & 0.6685 & \\
\hline $\mathrm{G}_{4}$ & 32.0563 & 0.1162 & 0.7226 & 0.9368 & 0.0100 & \\
\hline $\mathrm{G}_{1}$ & 43.2862 & 0.3769 & 0.6014 & 0.1388 & 0.0606 & \multirow{4}{*}{ Heavy } \\
\hline $\mathrm{G}_{2}$ & 23.9369 & 0.6120 & 0.3851 & 0.1756 & 0.0100 & \\
\hline $\mathrm{G}_{3}$ & 50.0 & 0.1461 & 0.3731 & 0.5570 & 0.0100 & \\
\hline $\mathrm{G}_{4}$ & 19.0656 & 0.3590 & 0.3761 & 0.5329 & 0.5408 & \\
\hline
\end{tabular}




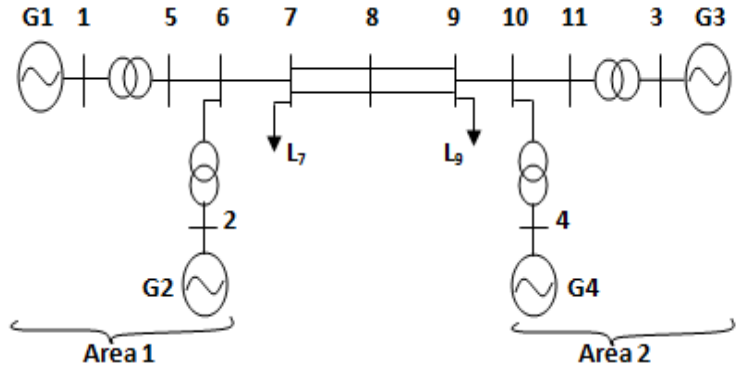

Fig.3: Four-Machine, Two Area System

Table 1: Loading conditions (in per unit)

\begin{tabular}{|c|c|c|c|c|c|c|}
\hline \multirow{2}{*}{ Load } & \multicolumn{2}{|c|}{ Nominal } & \multicolumn{2}{c|}{ Heavy } & \multicolumn{2}{c|}{ Light } \\
\cline { 2 - 7 } & $\mathrm{P}$ & $\mathrm{Q}$ & $\mathrm{P}$ & $\mathrm{Q}$ & $\mathrm{P}$ & $\mathrm{Q}$ \\
\hline $\mathrm{A}$ & 17.67 & -2.50 & 20.00 & -3.50 & 15.67 & -2.50 \\
\hline $\mathrm{B}$ & 9.67 & -1.00 & 9.67 & -1.00 & 9.67 & -1.00 \\
\hline
\end{tabular}

Table 2: Eigenvalues and damping ratios without PSS

\begin{tabular}{|l|l|l|}
\hline \multicolumn{1}{|c|}{ Nominal } & \multicolumn{1}{|c|}{ Heavy } & \multicolumn{1}{c|}{ Light } \\
\hline$-0.589 \pm 7.463 \mathrm{i}$, & $-89.88 \pm 0.061 \mathrm{i}, 1471.2$ & $\mathbf{0 . 0 9 1} \pm \mathbf{4 . 0 4 7 i}$, \\
0.078 & $\mathbf{0 . 1 2 7} \pm \mathbf{4 . 0 6 8 i}, \mathbf{- 0 . 0 3 1}$ & $\mathbf{- 0 . 0 2 2}$ \\
$-0.515 \pm 7.292 \mathrm{i}$, & $-0.343 \pm 7.656 \mathrm{i}, 0.044$ & $-0.706 \pm 7.303 \mathrm{i}$, \\
0.070 & $-0.514 \pm 7.303 \mathrm{i}, 0.070$ & 0.096 \\
$\mathbf{0 . 1 0 4} \pm \mathbf{4 . 0 5 1 i}$, & & $-0.535 \pm 7.278 \mathrm{i}$, \\
$\mathbf{- 0 . 0 2 5}$ & & 0.074 \\
\hline
\end{tabular}

Table 3: Eigenvalues and damping ratios with PSS

\begin{tabular}{|c|c|c|}
\hline Nominal & Heavy & Light \\
\hline$-31.59 \pm 40.93 \mathrm{i}, 0.772$ & $-41.88 \pm 20.94 \mathrm{i}, 2.00$ & $-29.01 \pm 41.70 \mathrm{i}, 0.696$ \\
\hline$-7.22 \pm 14.88 \mathrm{i}, 0.485$ & $-29.15 \pm 7.52 \mathrm{i}$ & $-5.95 \pm 13.43 \mathrm{i}, \quad 0.443$ \\
\hline$-5.83 \pm 10.26 \mathrm{i}, 0.568$ & 3.876 & $-7.34 \pm 10.32 \mathrm{i}, 0.711$ \\
\hline$-2.48 \pm 4.10 \mathrm{i}, \quad 0.605$ & $-4.99 \pm 11.33 \mathrm{i}$ & $-7.03 \pm 4.31 \mathrm{i}, \quad 1.631$ \\
\hline$-4.41 \pm 2.90 \mathrm{i}, \quad 1.521$ & 0.440 & $-1.78 \pm 2.58 \mathrm{i}, \quad 0.689$ \\
\hline$-1.55 \pm 1.99 \mathrm{i}, \quad 0.779$ & $-2.08 \pm 5.28 \mathrm{i}$ & $-2.04 \pm 0.23 i, \quad 8.869$ \\
\hline$-1.06 \pm 0.25 i, \quad 4.24$ & 0.394 & $-1.37 \pm 0.27 i, \quad 5.074$ \\
\hline & $\begin{array}{l}-1.62 \pm 3.69 \mathrm{i}, \\
0439\end{array}$ & $-1.10 \pm 0.09 \mathrm{i}$ \\
\hline & $-1.33 \pm 1.15 \mathrm{i}$ & \\
\hline & & \\
\hline
\end{tabular}

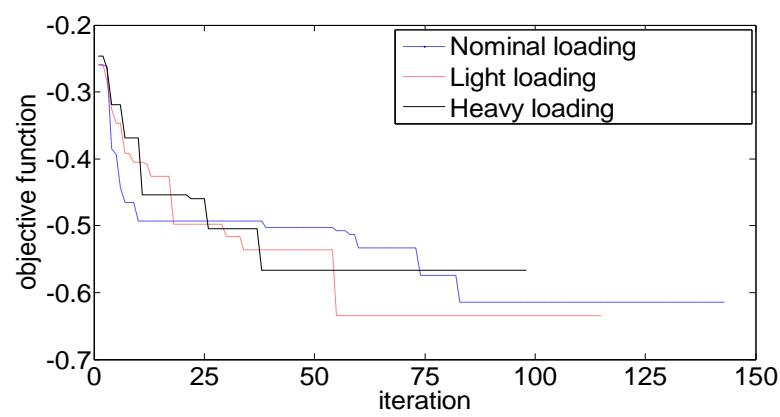

Fig.4: Variation of objective functions, J

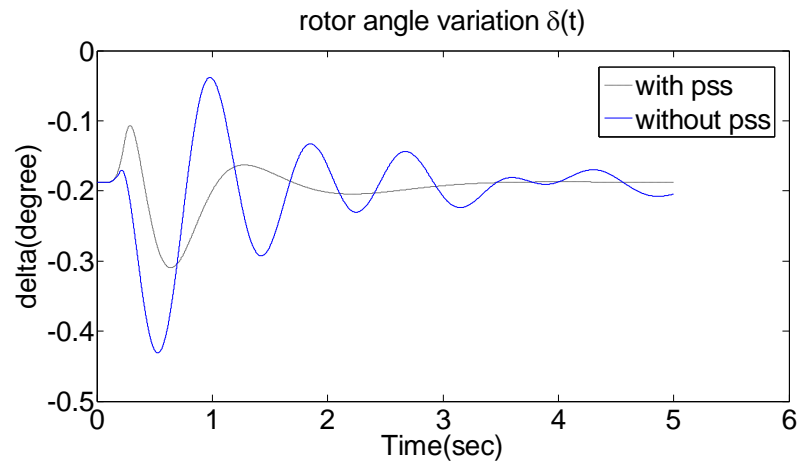

Fig.5: Rotor angle response in nominal loading for Gen2

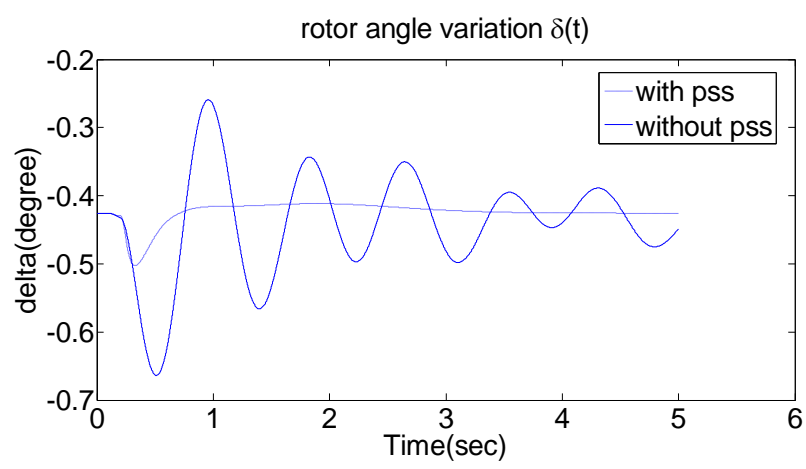

Fig.6: Rotor angle response in heavy loading for Gen2

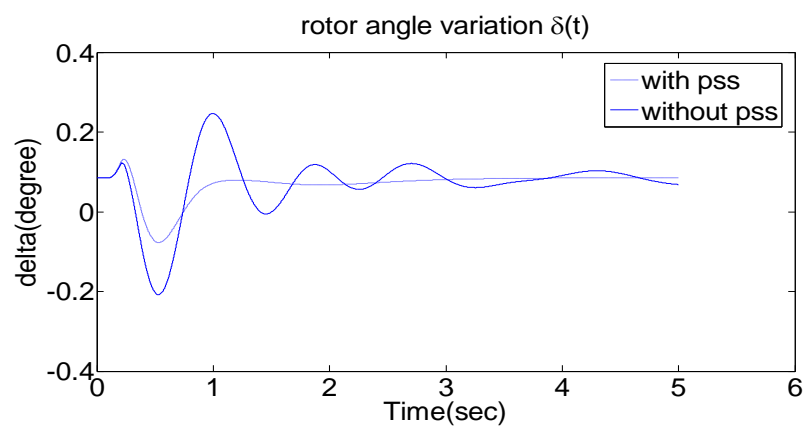

Fig.7: Rotor angle response in light loading for Gen2

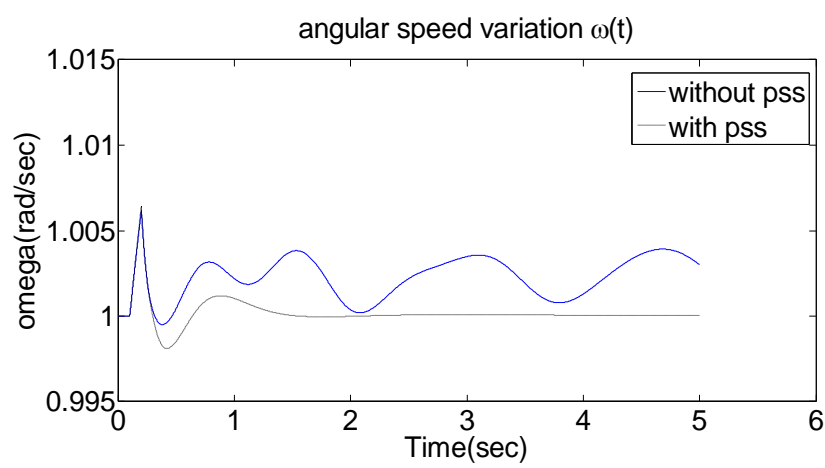

Fig.8: Rotor speed response in nominal loading for Gen2 


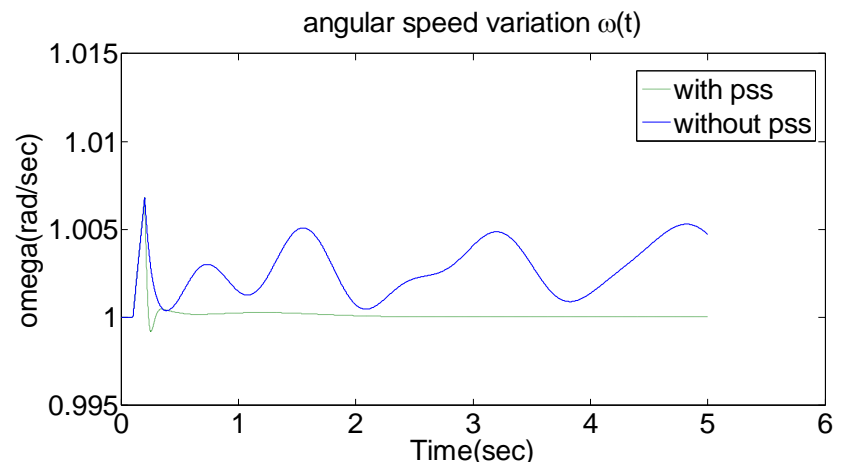

Fig.9: Rotor speed response in heavy loading for Gen2

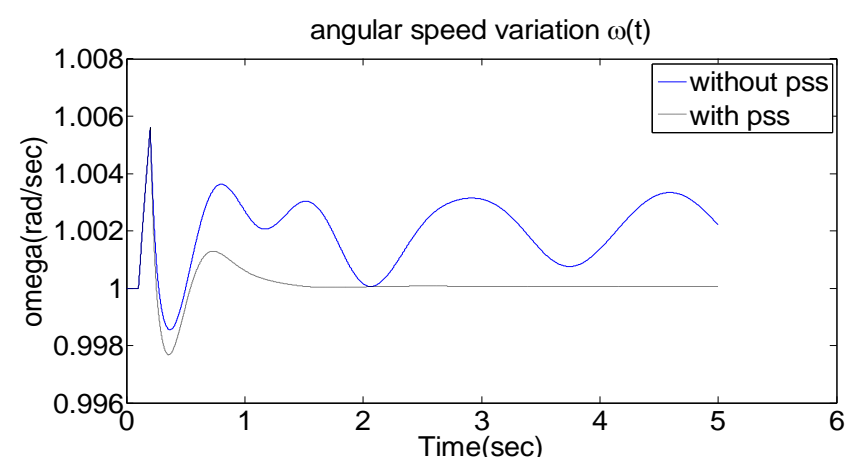

Fig.10: Rotor speed response in light loading for Gen2

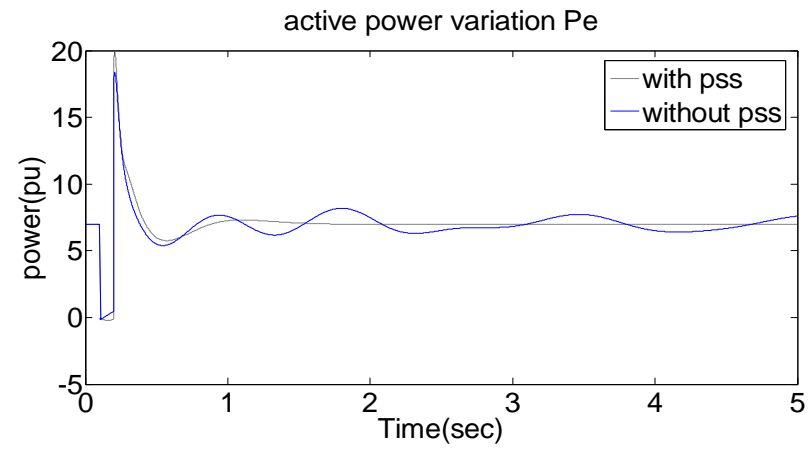

Fig.11: Active power output in nominal loading for Gen2

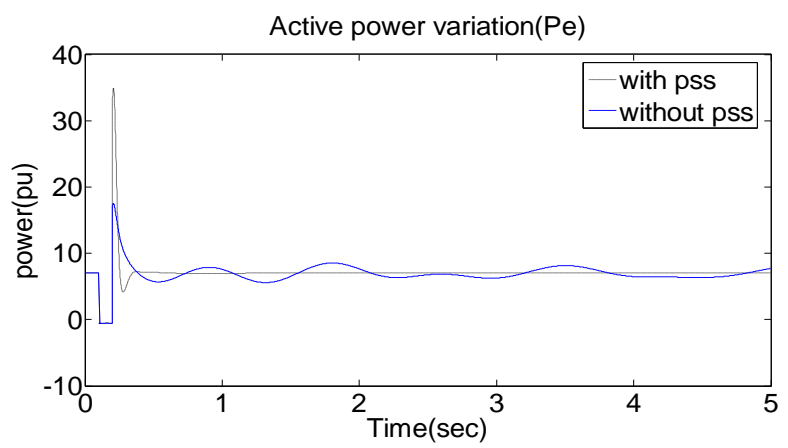

Fig.12: Active power output in heavy loading for Gen2

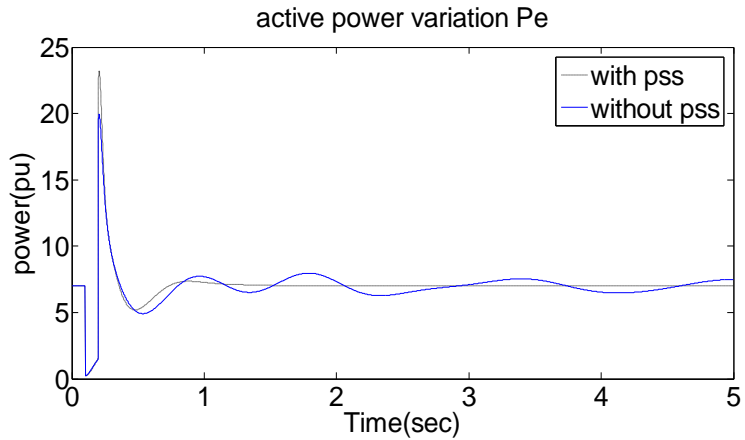

Fig.13: Active power output in light loading for Gen2

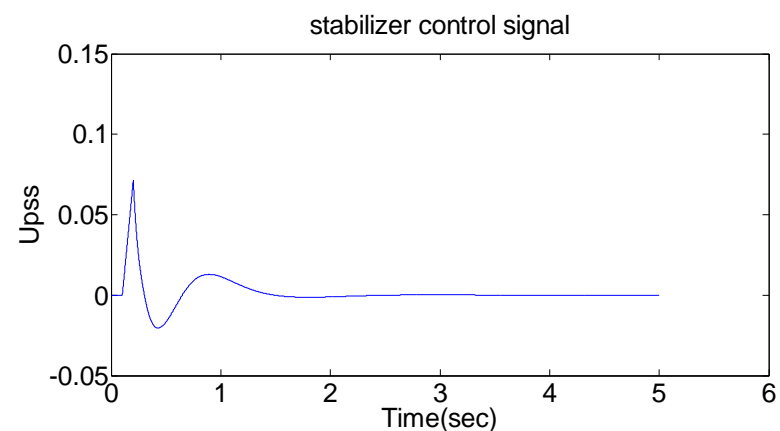

Fig.14: Stabilizer control signal (Upss) in nominal loading for Gen 2

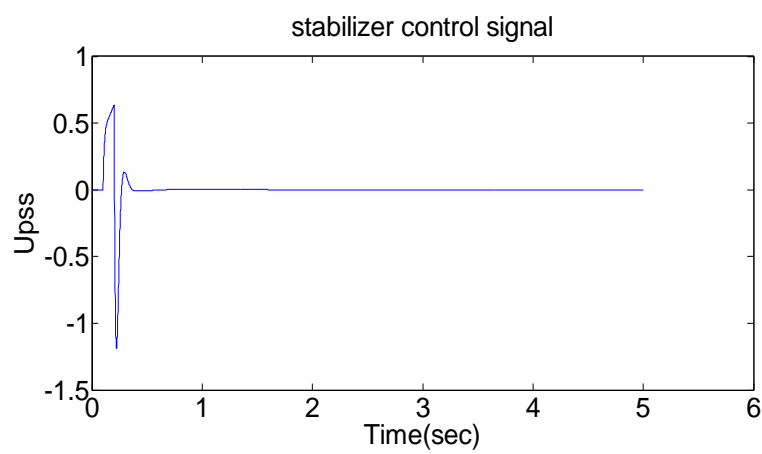

Fig.15: Stabilizer control signal (Upss) in heavy loading for Gen 2

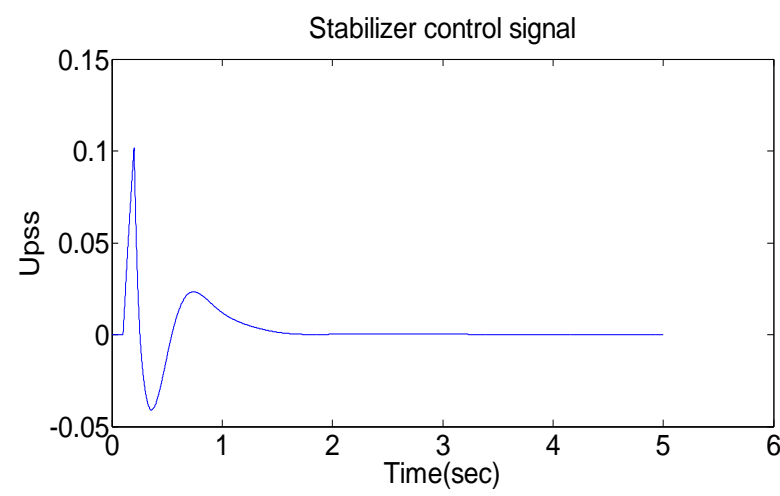

Fig.16: Stabilizer control signal (Upss) in heavy loading for Gen 2 
It is observed from the Table IV that the electromechanicalmode Eigen values have been shifted to the left in s-plane and the system damping has been enhanced and improved for the proposed IWO based PSS.

For further illustration a three phase fault disturbance at bus 7 at end of line 5-7 is considered for the non linear time simulations. To demonstrate the effectiveness and robustness of the proposed PSS different loading conditions has been considered. The rotor angle variations, angular speed variations, active power variations for nominal, heavy and light loading for Gen 2 are shown in Fig. 4-12.The stabilizer control signal response for above cases are also shown in Fig. 13-15.

\section{CONCLUSION}

In this paper the performance of the Invasive Weed Optimization technique is investigated for the design of Power System Stabilizers for the damping of low frequency oscillation of a three machine example power system. An eigenvalue value objective function is selected for optimizing the PSS performance. Both eigenvalue based and non-linear time domain simulation is carried out for three different loading conditions. The results show the effectiveness of IWO in tuning PSS for the damping of low frequency oscillations of a multi-machine power system.

\section{Appendix: Machine Model}

$$
\begin{aligned}
& \dot{\boldsymbol{\delta}}=\omega_{b}\left(\omega_{i-1}\right) \\
& \dot{\boldsymbol{\omega}}_{i}=\left(P_{m^{-}} P_{e i}-D_{i}\left(\omega_{i}-1\right)\right) / M_{i} \\
& \dot{\boldsymbol{E}}_{q i}^{\prime}=\left(\boldsymbol{E}_{f d i}-\left(\boldsymbol{x}_{d i}-\boldsymbol{x}_{d i}{ }_{d i}\right) i_{d i}-\boldsymbol{E}_{q i}\right) / \boldsymbol{T}_{d o i} \\
& \dot{\boldsymbol{E}}_{d i}^{\prime}=\left(\left(\boldsymbol{x}_{q i}-\boldsymbol{x}_{d i}{ }_{d i}\right) i_{q i}-\boldsymbol{E}_{d i}\right) / \boldsymbol{T}_{q o i} \\
& T_{e i}=E^{\prime}{ }_{q i} i_{q i}-\left(x_{q i}-x^{\prime}{ }_{d i}\right) i_{d i} i_{q i}
\end{aligned}
$$

$\begin{array}{ll}\delta & \text { rotor angle } \\ \omega & \text { rotor speed } \\ P_{m} & \text { mechanical input power } \\ P_{e} & \text { electrical output power } \\ E_{q}, & \text { internal voltage behind } x_{d i} \\ E_{f d} & \text { equivalent excitation voltage } \\ T_{e} & \text { electric torque } \\ T_{d o} & \text { time constant of excitation circuit } \\ T_{q o} & \text { regulator time constant } \\ v_{r e f} & \text { reference voltage } \\ v & \text { terminal voltage }\end{array}$

\section{REFERRENCES}

[1] Kundur P., Power System Stability And Control (McGraw-Hill Press, 1994)

[2] Anderson P. M., Fouad, A.A., Power System Control and Stability, IEEE series on power engineering, $2^{\text {nd }}$ edition.

[3] Larsen E. and Swann D., Applying power system stabilizers, IEEE Trans. Power App. Systems, Vol. PAS100, 1981, pp.3017-3046.

[4] Cao Y., Jiang L., Cheng S., Chen D., Malik O.P. and Hope G.S., " A nonlinear variable structure stabilizer for power system stability", IEEE Trans. Energy Conversion, vol. 9, Sept. 1994,pp. 489-495.

[5] Hiyama T. and Sameshima T., "Fuzzy logic control scheme for on-line stabilization of multimachine power system,” Fuzzy Sets Sys., vol. 39, 1991 pp. 181-194.

[6] Abido M.A. and Abdel-Magid Y.L., "A hybrid neurofuzzy power system stabilizer for multi-machine power systems," IEEE Trans. Power Syst., vol. 13, Nov. 1998pp. 1323-1330.

[7] D. Xia and G. T. Heydt, "Self-tuning controller for generator excitation control," IEEE Trans. Power App. Syst., 1983, pp. 1877-1885.

[8] P. Kundur, M. Klein, G. J. Rogers, and M. S. Zywno, Application of power system stabilizers for enhancement of overall system stability, IEEE Trans. on Power Systems. Vol. PAS-108, 1989, pp.614-626.

[9] R. J. Fleming, M. A. Mohan, K Parvatism, “ Selection of parameters of stabilizers in multi-machine power systems", IEEE Trans, PAS, vol. 100, no. 5,1981, pp. 2329-2333.

[10] Mahdiyeh Eslami, Hussain Shareef and Azah Mohamed, "Optimal tuning of power system stabilizers using modified particle swarm optimization", Proceedings of the 14th International Middle East Power Systems Conference (MEPCON'10), Cairo University, Egypt, December 19-21, 2010.

[11] M. A. Abido, "A novel approach to conventional power system stabilizer design using tabu search," Int. J. Electr. Power Energy Syst., vol. 21, 1999, pp. 443-454.

[12] Y. L. Abdel-Magid and M. A. Abido, "Optimal multiobjective design of robust power system stabilizers using genetic algorithms," IEEE Transactions on Power Systems, vol. 18, 2003,pp. 1125-1132.

[13] A. L. B. Do Bomfim, et al., "Simultaneous tuning of power system damping controllers usinggenetic algorithms," IEEE Trans. Power Syst.,vol. 15,2000, pp. 163-169.

[14] M. A. Abido, Robust design of multi-machine power system stabilizers using simulated annealing, IEEE Trans. on Energy Conversion, Vol.15, No. 3, 2003, pp. 297-304.

[15] M. A. Abido, Y. L. Abdel-Magid, Optimal design of power system stabilizers using evolutionary programming, IEEE Trans. on Energy Conversion, Vol.17, No. 4,2002, pp.429-436.

[16] P. Zhang and A. H. Coonick, "Coordinated synthesis of PSS parameters in multi-machine powersystems using the method of inequalities applied to genetic algorithms," IEEE Trans. Power Syst., vol. 15, 2000, pp. 811-816.

[17] A.R. Mehrabian, C. Lucas, A novel Numerical Optimization Algorithm Inspired from Weed Colonization, Ecological Informatics, 2006, vol 1.pp-355366. 
[18] A.R. Mehrabian, A. Yousefi-Koma, Optimal Positioning of Piezoelectric Actuators on a Smart Fin using Bioinspired Algorithms, Aerospace Science and Technology,2007, vol 11, pp 174-182.

[19] A. R. Mallahzadeh ,S. Es'haghi, A Alipour, “ Design of an E shaped MIMO Antenna using IWO Algorithm for Wireless Application at 5.8 Ghz", Progress in Electromagnetic Research,PIER 90, 2009,187-203.

[20] X. Zhang, Y. Wang, G. Cui, Y. Niu, J. Xu, Application of a novel IWO to the design of encoding sequence for DNA computing, Comput. Math. Appl. 57, Jun 2009, pp. 2001-2008,

[21] H. Sepehri Rad , C. Lucas, “ A Recommender System based on Invasive Weed Optimization Algorithm", IEEE
Congress on Evolutionary Computation, CEC 2007,pp 4297-4304.

[22] M. Shaheri-Ardakani, M. Rshanaei, A. Rahimi-Kian, C. Lucas, "A study of electricity market dynamics using Invasive Weed Colonization Optimization," in Proc. IEEE Symp. Comput.Intell. Games, 2008, pp.276-282.

[23] P. W. Sauer and M. A. Pai, Power System Dynamics and Stability.(Englewood Cliffs, NJ: Prentice-Hall, 1998.)

[24] S. Karimkashi, Ahmed A. Kishk, "Invasive Weed Optimization and its Features in Electromagnetics", IEEE Transactions on Antenna and Propagation, Vol. 58, No. 4, April 2010, pp. 1269-1278 